\title{
Adversidade na infância prediz sintomas depressivos e tentativas de suicídio em mulheres adultas portuguesas
}

\author{
Adversity in childhood predicts depressive \\ symptoms and suicide attempts in \\ adult Portuguese women
}

\author{
Vanessa Cristina Pires PINTO' \\ Joana Ferreira Cardoso ALVES' \\ Ângela Costa MAIA ${ }^{1}$
}

\begin{abstract}
Resumo
Este estudo examinou a prevalência de 10 categorias de experiências adversas na infância autorrelatadas em mulheres adultas portuguesas, e avaliou se essas experiências eram preditoras do índice de sintomatologia depressiva e de tentativas de suicídio. Um total de 225 mulheres completou o Adverse Childhood Experiences Study Questionnaire e a subescala de depressão do Inventário de Sintomas Psicopatológicos. Quase 96,0\% das mulheres relatou ter sido exposta a pelo menos uma experiência adversa na infância e adolescência. Os resultados da regressão linear indicam que a adversidade total explica $6,6 \%$ da variância dos sintomas de depressão, enquanto a regressão logística mostra que o incremento de um ponto na adversidade total aumenta o risco de tentativas de suicídio em 1818 vezes. Os resultados deste estudo mostram que a exposição a experiências adversas na infância é frequente e o seu grau é preditor de sintomatologia depressiva e tentativas de suicídio.
\end{abstract}

Palavras-chave: Depressão; Experiências adversas; Suicídio.

\begin{abstract}
This study examined the prevalence of 10 categories of self-reported childhood adverse experiences in adult Portuguese women and assessed whether these factors were predictive of depressive symptoms and suicide attempts. A total of 225 women completed the Adverse Childhood Experiences Study Questionnaire and evaluated the depression subscale of the Brief Symptoms Inventory. Almost 96.0\% of the participant women reported being exposed to at least one adverse experience in childhood and adolescence. Linear regression indicated that the total adversity explains $6.6 \%$ of the variance in symptoms of depression, whereas logistic regression indicated that the increase in adversity by one point increased the risk of suicide attempts 1,818 times. The results obtained show that exposure to adverse childhood experiences is frequent and that the degree of exposure is a predictor of depressive symptoms and suicide attempts.
\end{abstract}

Keywords: Depression; Adverse experiences; Suicide.

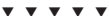

1 Universidade do Minho, Escola de Psicologia. Largo do Paço, 4704-553, Braga, Portugal. Correspondência para/Correspondence to: A.C. MAIA. E-mail: <angelam@psi.uminho.pt>

Artigo baseado na dissertação de V.C.P. PINTO, intitulada "Adversity, depressive symptoms and suicide attempts: A comparison between women in prison and women in the community". Universidade do Minho, 2013.
} 
As experiências adversas na infância são altamente prevalentes e tendem a coocorrer, sendo a exposição a múltiplos tipos de experiências adversas um dado consistente na literatura (Afifi, Mota, Dasiewicz, MacMillan, \& Sareen, 2012; Edwards, Holden, Felitti, \& Anda, 2003; Felitti et al., 1998; Lu, Mueser, Rosenberg, \& Jankowski, 2008). O estudo da relação entre as experiências adversas na infância e o estado de saúde na idade adulta, tem verificado que a adversidade está associada a consequências negativas em diferentes dimensões, que incluem mais problemas físicos, psicológicos, cognitivos, comportamentais, sociais e emocionais (Afifi et al., 2012; Azevedo \& Maia, 2006; Nurius, Logan-Greene, \& Green, 2012; Widom, Czaja, Bentley, \& Johnson, 2012). O aumento do risco de desenvolvimento de transtornos do foro psíquico, nomeadamente depressão e tentativas de suicídio, está entre um dos resultados mais consistentes. Por exemplo, estudos têm verificado que existe uma relação significativa entre experiências adversas e sintomatologia depressiva (Mullen, Martin, Anderson, Romans, \& Herbison, 1996; MacMillan et al., 2001). Um dos mais conhecidos, o Adverse Childhood Experiences Study (ACE Study), de Felitti et al. (1998), recorreu a uma amostra de mais de 17 mil pessoas, constatando que a prevalência dos sintomas depressivos no ano anterior à avaliação era proporcional à história de exposição às experiências adversas. Os resultados do ACE Study indicam que os indivíduos sujeitos a quatro ou mais tipos de experiências adversas na infância ou adolescência têm mais $460 \%$ de probabilidade de vir a sofrer de depressão (Felitti, 2002). A relação positiva entre a adversidade e a depressão é empiricamente consensual, tendo-se verificado sistematicamente que quanto maior o número de experiências adversas vivenciadas, maior o risco de se desenvolver uma perturbação depressiva (Anda et al., 2006; Chapman et al., 2004; Dube, Felitti, Dong, Giles, \& Anda, 2003). Além disso, seu grau de severidade também aumenta (Lu et al., 2008).

As investigações são também consistentes quanto ao fato de as experiências adversas constituírem um fator de risco para tentativas de suicídio. No âmbito do ACE Study, foi observado que a dio, uma vez que quem experienciava sete ou mais de suas categorias, tinha a probabilidade de tentar suicídio aumentada em 31,1 vezes (Dube et al., 2001). O estudo de Silva e Maia (2010), com uma amostra de 100 adultos com obesidade mórbida, evidenciou que as tentativas de suicídio eram prevalentes nessa população, e constatou que estavam relacionadas com o relato de adversidade na infância, sendo o total delas um preditor significativo de tentativas de suicídio.

Em suma, a literatura sugere que o impacto das experiências adversas na infância sobre o índice de depressão e tentativas suicidas é significativo, cumulativo e destrutivo (Felitti, 2002; Felitti et al., 1998), e alguns estudos têm procurado averiguar quais dessas experiências mais contribuem para o transtorno mental posterior. Por exemplo, um estudo muito recente, de Nurius et al. (2012), verificou que as mais nefastas são transtorno mental parental, abuso emocional e abuso físico. Este último foi também identificado por Afifi et al. (2012), ao usarem uma amostra americana ( $n=34$ 653), como sendo associado a transtornos de humor e de ansiedade, transtornos relacionados ao uso de substâncias e de distúrbios de personalidade. Neste último trabalho, o autor concluiu que a eliminação dessa prática traria uma diminuição de 2 a $5 \%$ nos transtornos do eixo I e de 4 a $7 \%$ nos transtornos do eixo II. Em relação a experiências concretas relacionadas com tentativas de suicídio, Fuller-Thomson e Dalton (2011) verificaram que adultos que vivenciaram na infância divórcio parental têm maior probabilidade de desenvolver ideação suicida. Nas mulheres essa associação deixou de ser significativa quando outros tipos de adversidade foram incluídas nas análises, sugerindo que outras experiências adversas coocorrentes têm maior impacto do que o divórcio entre os pais.

No entanto, de forma discordante, um outro estudo evidenciou que a vivência de abuso na infância combinado com divórcio parental aumentava a probabilidade de ideação e tentativa de suicídio em comparação com os indivíduos que foram expostos apenas ao divórcio entre os pais ou a outro tipo de adversidade. Dessa forma, conclui que tanto o divórcio quanto outras experiências adversas estão 
associados à ideação e tentativas de suicídio (Afifi, Boman, Fleisher, \& Sareen, 2009).

Apesar da consistência dos resultados sobre a relação entre vitimação na infância e transtorno mental na idade adulta, alguns autores (Edwards et al., 2003) têm alertado para o fato da maioria das investigações centrar-se em apenas um ou dois tipos de experiências adversas. Por essa razão, neste estudo analisamos 10 categorias de adversidade: 5 contra o indivíduo (abuso emocional, físico, sexual, negligência física e emocional) e 5 do ambiente familiar (familiares consumidores de álcool e/ou drogas, transtorno mental ou suicídio na história familiar, prisão de um familiar, violência doméstica contra a figura materna e divórcio entre os pais). Assim, espera-se fazer uma caracterização mais abrangente das experiências adversas e evitar que se atribua, de forma errônea, a influência a um único tipo de abuso (Felitti et al., 1998), bem como para não ignorar a evidência de exposição a outras formas de adversidade (Rodgers et al., 2004).

Devido ao fato de as mulheres reportarem mais que os homens a exposição a experiências adversas na infância e tentativas de suicídio (Dube et al., 2001; Dube et al., 2003; Edwards et al., 2003), além da associação entre adversidade e depressão também ser mais forte entre elas (MacMillan et al., 2001) e existir maior vulnerabilidade e morbidade psicológica, este estudo foca-se no sexo feminino. Até o momento, nenhuma investigação explorou esse fenômeno apenas em mulheres analisando um total de 10 tipos de adversidade.

Dessa forma, os objetivos deste trabalho são caracterizar a prevalência das experiências adversas autorrelatadas em mulheres adultas portuguesas e avaliar se essas experiências estão relacionadas a sintomas depressivos e tentativas de suicídio. Além disso, buscou-se analisar especificamente a contribuição de cada tipo de experiência para as variáveis dependentes estudadas. De acordo com a literatura prevê-se: 1) uma elevada prevalência de experiências adversas relatadas; 2 ) uma relação significativa entre experiências adversas na infância e índice de sintomatologia depressiva na idade adulta; e 3) que a adversidade seja um preditor de sintomatologia depressiva e tentativas de suicídio.

\section{Método}

\section{Participantes}

A amostra foi constituída por 225 mulheres com idades compreendidas entre 18 e 78 anos (Média - $M=36,30$, Desvio-Padrão - $D P=11,506$ ), numa amostragem de conveniência, as quais foram recrutadas entre usuárias e profissionais de centros de qualificação profissional e outras instituições de apoio social. Os critérios de inclusão que caracterizaram as participantes foram: serem mulheres adultas residentes no Norte do país e que compreendessem o português, sendo que a maioria das delas pertencia a um grupo socioeconômico baixo. As pessoas que aceitaram participar durante o período do estudo também foram incluídas na amostra. Apenas oito pessoas recusaram o convite referindo falta de tempo. Outras características sociodemográficas estão descritas na Tabela 1.

\section{Instrumentos}

Um Questionário Sociodemográfico foi usado para recolher informações acerca da idade,

Tabela 1

Características demográficas das participantes. Portugal, 2012

\begin{tabular}{lcc}
\hline Categorias & $\mathrm{n}$ & $\%$ \\
\hline Estado civil & & \\
Casada ou amasiada & 137 & 61.7 \\
Solteira & 55 & 24.8 \\
Divorciada ou separada & 23 & 10.4 \\
Viúva & 7 & 3.2 \\
Escolaridade & & \\
Nunca foi à escola & 2 & 0.9 \\
Menos de 4 anos & 5 & 2.2 \\
$1^{\circ}$ ciclo & 31 & 13.8 \\
$2^{\circ}$ ciclo & 57 & 25.4 \\
$3^{\circ}$ ciclo & 57 & 25.4 \\
Ensino secundário & 30 & 13.4 \\
Formação universitária & 42 & 18.8 \\
Situação profissional & & \\
Empregada & 122 & 56.5 \\
Desempregada & 94 & 43.5 \\
\hline
\end{tabular}

Nota: Em Portugal, o Ensino é dividido da seguinte forma: Ensino básico, que inclui o $1^{\circ}$ ciclo ( $1^{\circ}$ ao $4^{\circ}$ ano), $2^{\circ}$ ciclo ( $5^{\circ}$ e $6^{\circ}$ ano ) e $3^{\circ}$ ciclo $\left(7^{\circ}\right.$ ao $9^{\circ}$ ano), sendo equivalente ao Ensino Fundamental no Brasil; e Ensino secundário $\left(10^{\circ}, 11^{\circ}\right.$ e $12^{\circ}$ ano), sendo equivalente ao Ensino Médio no Brasil. 
estado civil, escolaridade e situação profissional das participantes, além disso, foi utilizada a versão portuguesa do Adverse Childhood Experiences (ACE) Study Questionnaire (Felitti et al., 1998; Silva \& Maia, 2008), a qual inclui questões de autorrelato acerca de 10 categorias de experiências adversas divididas em duas áreas: experiências sobre as quais a criança é vítima direta (abuso emocional, físico e sexual e negligência física e emocional) e experiências integradas num contexto familiar disfuncional (familiares consumidores de substâncias, transtorno mental ou suicídio na família, prisão de um membro familiar, violência doméstica contra a figura materna e divórcio entre os pais).

Para cada uma dessas áreas existe um conjunto de questões que permite avaliar a ocorrência da experiência, sendo que a resposta é cotada com valor 0 (zero) ou 1 (um). As questões de abuso físico e emocional e de negligência só são cotadas com 1 quando a resposta é "muitas vezes" ou "muitíssimas vezes". Para cada categoria, a participante era definida como sendo vítima de uma experiência adversa se pontuasse positivamente em um de seus itens.

Esse questionário possibilita calcular o Índice Total de Adversidade. Este corresponde ao somatório dos valores obtidos por cada indivíduo nas 10 categorias, podendo variar de zero (quando não há relato de qualquer experiência adversa) a 10 (quando existe o relato de adversidade de todos os tipos). Estudos de consistência temporal mostram que a versão portuguesa apresenta valores adequados (Pinto, Correia, \& Maia, 2014; Silva \& Maia, 2013).

O Brief Symptoms Inventory (BSI, Inventário de Sintomas Psicopatológicos) (Derogatis, 1982; versão portuguesa de Canavarro, 1999) foi utilizado para avaliar a sintomatologia psicológica na última semana. Ele é composto por nove subescalas que avaliam diferentes dimensões psicopatológicas (Somatização, Obsessões-Compulsões, Sensibilidade Interpessoal, Depressão, Ansiedade, Hostilidade, Ansiedade Fóbica, Ideação Paranoide e Psicoticismo); no entanto, neste estudo foi utilizada so- itens organizados numa escala tipo Likert em que a resposta varia de "nunca" a "muitíssimas vezes". A adaptação portuguesa mostra que o instrumento tem boas características psicométricas. No nosso estudo a consistência interna (alpha de Cronbach) dessa escala foi de 0,848 . As tentativas de suicídio foram medidas através da questão "Alguma vez se tentou suicidar?".

\section{Procedimentos}

Inicialmente foi pedida autorização à Comissão de Ética para a realização deste estudo, tendo sido obtida sob o Protocolo CA_CIPsi_082012. A coleta de dados ocorreu em Centros de Novas Oportunidades, onde as participantes estavam em processo de formação, e entre usuárias e profissionais de vários serviços de apoio social localizados no norte do país. Nos casos de participantes com baixa escolaridade recorreu-se ao relato de uma terceira pessoa. Em todos os casos explicitou-se a garantia de que a informação recolhida permaneceria anônima e confidencial colhendo, também, a assinatura de Termo de Consentimento. As informações foram recolhidas entre novembro de 2008 e outubro de 2012.

Todos os dados foram introduzidos e analisados no software Statistical Package for the Social Sciences (SPSS, versão 19.0). Recorreu-se à estatística descritiva para caracterizar os participantes em termos sociodemográficos, bem como para obter a frequência e percentagem das diferentes categorias de adversidade.

A análise exploratória dos dados revelou não terem sido cumpridos os pressupostos necessários à utilização de testes paramétricos. No entanto, estes foram utilizados, juntamente com os equivalentes não paramétricos, como sugerido por Fife-Schaw (2006). Como as conclusões de ambos foram iguais em todos os casos, optou-se por apresentar os resultados dos testes paramétricos devido à sua maior robustez e da possibilidade de uso de análises multivariadas, reduzindo, assim, o número de testes realizados e, consequentemente, o erro do Tipo I. 
Foi efetuado um teste $t$ em uma amostra a fim de analisar as diferenças existentes entre as participantes e a população portuguesa no que diz respeito à sintomatologia depressiva. A regressão linear hierárquica foi utilizada para analisar se as experiências adversas são preditores desta sintomatologia, controlando o nível educacional. A significância do modelo foi testada tendo por base o $R^{2}$ change statistics. Os testes Qui-quadrado foram usados para avaliar a associação entre a variável "tentativas de suicídio" e diferentes categorias de experiências adversas. Para analisar o poder preditivo da adversidade total para as tentativas de suicídio efetuou-se uma análise de regressão logística.

\section{Resultados}

Verificou-se que 213 (95,9\%) das mulheres estudadas revelaram ter vivido pelo menos uma experiência adversa na infância, sendo que destas, $38(17,1 \%)$ disseram ter experienciado 5 ou mais tipos de adversidade. A média de experiências foi de 2,92 $(D P=1,76)$. A frequência de cada uma das categorias de adversidade encontra-se na Tabela 2. A média da sintomatologia depressiva foi 0,95 $(D P=0,87)$ e 17 participantes $(7,6 \%)$ indicaram já ter tentado o suicídio.

Com relação à média dos sintomas depressivos, a realização de um teste $t$ para uma amostra,

Tabela 2

Frequência e percentagem de cada uma das categorias de adversidade. Portugal, 2012

\begin{tabular}{lcc}
\hline Categorias & $\mathrm{n}$ & $\%$ \\
\hline Abuso emocional & 76 & 33.8 \\
Abuso físico & 104 & 46.2 \\
Abuso sexual & 20 & 8.9 \\
Negligência física & 62 & 27.6 \\
Negligência emocional & 85 & 37.9 \\
Familiares consumidores & 51 & 22.7 \\
Transtorno mental e/ou tentativa de suicídio familiar & 55 & 24.4 \\
Prisão de familiares & 10 & 4.4 \\
Violência doméstica & 178 & 79.8 \\
Divórcio dos pais & 13 & 5.8 \\
\hline
\end{tabular}

considerando os dados da adaptação portuguesa (Canavarro, 1999), permitiu verificar que não há diferenças significativas em relação à população geral $(M=0,946 ; D P=0,870$ vs $M=0,893$; $D P=0,722), t_{(218)}=0,901, p=0,369$. Verificou-se que a adversidade total estava positivamente relacionada com os sintomas depressivos $(r=0,289$, $p<0,001)$. Apesar da ampla variação de idade das participantes, verificou-se não existir associação entre a mesma e sintomas depressivos, $r=0,021$, $p=0,760$. Levando em consideração que um grande número de participantes estava desempregada, foi avaliado se havia diferenças no que diz respeito à sintomatologia depressiva em função da situação profissional e verificou-se que estas não existiam, $t_{(208)}=-1,007, p=0,315$. O mesmo não aconteceu com o nível de escolaridade, $F_{(6,214)}=3,209$, $p=0,005$. Frente a esses resultados, realizou-se uma regressão hierárquica para analisar a contribuição da totalidade da adversidade controlando o nível de escolaridade, tendo verificado que o modelo explicava 6,6\% da variância dos sintomas depressivos, sendo significativo, $F_{(1,212)}=15,555, p<0,001$, $t=3,944, p<0,001$. O $\beta$ da adversidade foi de 0,264

Com relação a cada tipo de adversidade, as mulheres que relataram abuso emocional $\left(t_{(217)}=-2,894, p=0,004\right)$, abuso físico $\left(t_{(217)}=\right.$ $4,417, p<0,001)$ ou negligência emocional $\left(t_{(216)}=\right.$ $-2,558, p=0,011)$, tinham familiares consumidores de substâncias $\left(t_{(217)}=-2,227, p=0,027\right)$ ou com transtorno mental $\left(t_{(217)}=-2,513, p=0,013\right)$, apresentavam maior sintomatologia depressiva em comparação com as que não foram expostas a essas experiências adversas. No que diz respeito às tentativas de suicídio, verificou-se que as participantes que as relataram apresentavam maior exposição à adversidade total em comparação com as que não tiveram atos suicidas $\left(t_{(220)}=-5,214, p<0,001\right)$.

Constatou-se também que as tentativas de suicídio estavam associadas ao abuso emocional $\left(\chi_{(1)}^{2}=5,157, p=0,023\right)$, sexual $\left(\chi_{(1)}^{2}=15,832, p<\right.$ $0,001)$, negligência física $\left(\chi_{(1)}^{2}=21,878, p<0,001\right)$, familiares consumidores de álcool e/ou drogas $\left(\chi^{2}{ }_{(1)}=13,715, p<0,001\right)$ e transtorno mental na história familiar $\left(\chi_{(1)}^{2}=5,092, p=0,024\right)$. A 
regressão logística, usando como preditor a adversidade total e controlando a sintomatologia depressiva, evidenciou que o modelo obtido é significativo, $\chi_{(1)}^{2}=14,579, p<0,001$, sendo que o aumento de um ponto na adversidade total aumenta o risco de tentativas de suicídio em 1818 vezes.

\section{Discussão}

Este estudo procurou verificar a prevalência de experiências adversas autorrelatadas em mulheres adultas e analisar a sua conexão com sintomatologia depressiva e tentativas de suicídio. Os resultados sugerem uma elevada prevalência de experiências adversas autorrelatadas e verificou-se que estas estão associadas ao índice de sintomatologia depressiva atual e tentativas de suicídio. Esses resultados corroboram com as hipóteses inicialmente colocadas. Os resultados são consistentes com estudos anteriores os quais demonstram que as experiências adversas são altamente prevalentes e coocorrentes (Anda et al., 2006; Edwards et al., 2003; Felitti, 2002; Lu et al., 2008).

O presente estudo está de acordo com os resultados encontrados por Lu et al. (2008), pois os tipos de adversidade mais relatados foram violência doméstica e abuso físico. No entanto, a prevalência das diversas categorias de adversidade que foram reportadas apresenta-se superior à encontrada em estudos anteriores. Isso pode decorrer do fato de o sexo feminino relatar um maior número de experiências adversas (Edwards et al., 2003) e como outros estudos, em sua maioria, apresentam amostras mistas, o relato não é tão expressivo como aquele aqui encontrado.

Foi evidenciado que o abuso emocional, a presença de consumo de substâncias e de transtorno mental na história familiar são os tipos de adversidade que permitem distinguir as mulheres com relação a sintomatologia depressiva e, simultaneamente, encontram-se relacionados com as tentativas de suicídio. Esses dados estão de acordo com o que foi encontrado por Nurius et al. (2012), os quais sugerem que o abuso emocional e o transtorno mental na família são os tipos de adversidade que mais efeitos negativos trazem no que tange à psicopatologia.

Por outro lado, verificou-se ainda que, a situação profissional não se encontra associada aos os sintomas depressivos. Uma explicação possível pode ser o fato de as mulheres desempregadas que participaram do presente estudo encontrarem-se em processo de formação em Centros de Novas Oportunidades, ou seja, estarem ativas em termos de formação e enriquecimento pessoal.

De acordo com resultados encontrados em outras pesquisas, este estudo permite concluir que a adversidade na infância está associada aos sintomas depressivos na idade adulta (Afifi et al., 2012; Edwards et al., 2003; Felitti et al., 1998; Lu et al., 2008) sendo um preditor significativo desses sintomas (Anda et al., 2006; Dube et al., 2003; LaNoue, Graeber, Hernandez, Warner, \& Helitzer, 2012). O fato da adversidade ser preditora de tentativas de suicídio também foi encontrado através de investigação empírica já existente (Dube et al., 2003; Felitti et al., 1998). Dube et al. (2001), mostraram que um acréscimo de uma unidade na adversidade aumenta a possibilidade de tentativa de suicídio em $60 \%$. Os nossos resultados apresentam expressão idêntica aos encontrados por Silva e Maia (2010), que demonstraram que as tentativas de suicídio tinham um risco aumentado em 2026 vezes por cada acréscimo de um ponto na totalidade da adversidade. Esses dados também estão de acordo com o encontrado por Fuller-Thomson e Dalton (2011) o qual evidencia que o divórcio parental não está associado com as tentativas suicidas.

A relação entre adversidade, sintomas depressivos e tentativas de suicídio pode ser explicada através de diversos mecanismos. Alguns autores sugerem que essa relação pode resultar não das experiências adversas em si, mas de fatores de risco que thes estão associados, como pobreza, ausência de apoio social etc. (Dube et al., 2003; MacMillan et al., 2001). Um outro modelo sugere que as experiências de adversidade se relacionam com um conjunto de características, como desregulação emocional, baixa autoestima, vinculação insegura ou dificuldades interpessoais, e que são essas características que constituem fragilidades para o desen- 
volvimento posterior de depressão e tentativas de suicídio, podendo atuar como mediadores nessa relação (Dube et al., 2001; Figueiredo, Fernandes, Matos, \& Maia, 2002; Whiffen, Thompson, \& Aube, 2000).

Estudos da neurociência têm apontado outra explicação, evidenciando que as experiências adversas são uma condição ambiental externa que traz diversas mudanças biológicas. Quando essas experiências são repetidas na infância o sistema de estresse está continuamente ativo, afetando o sistema límbico e o córtex pré-frontal medial (Anda et al., 2006; Dube et al., 2003; Maia, 2010). Dessa forma, experiências repetidas de adversidade potenciam alterações neuroanatômicas (estrutura cerebral) e neurofisiológicas (funcionamento cerebral) que acompanham permanentemente o indivíduo (Anda et al., 2006).

Este estudo é relevante pois é necessária uma maior compreensão desse fenômeno para poder ajustar e adequar os programas de prevenção e intervenção. Tendo em conta a prevalência e o impacto dessas experiências, torna-se fundamental desenvolver estratégias de prevenção primária, secundária e terciária (Azevedo \& Maia, 2006; Felitti et al., 1998) de forma a melhorar o estado de saúde das mulheres e a prevenir o suicídio (Dube et al., 2001).

Os programas de prevenção e de intervenção devem ser promovidos em diferentes contextos socioeconômicos, no entanto, tornam-se particularmente importantes nas populações mais vulneráveis e necessitadas. O objetivo é o de diminuir a incidência da adversidade e as suas consequências e, simultaneamente, fornecer e potencializar as capacidades da população para prolongar as mudanças obtidas a longo prazo, tornando-a autônoma. Assim, intervenções são necessárias para promover uma melhoria na saúde comunitária e, por outro lado, quebrar o ciclo intergeracional. Esse ciclo sugere que pais com problemas sociais, físicos, emocionais e psíquicos expõem os filhos a experiências adversas precoces, e se estas são preditivas da psicopatologia, então prevê-se que essas crianças sofrerão mais tarde de perturbações psíquicas, dando continuidade ao ciclo intergeracional quando do nascimento de seus filhos. As estratégias adotadas devem ser individuais, comunitárias, multidisciplinares e multidimensionais (Larkin, Shields, \& Anda, 2012; Nurius et al., 2012) refletindo uma conceitualização ecossistêmica em que todos os fatores de risco e proteção são considerados (Azevedo \& Maia, 2006).

Os resultados encontrados indicam que a exposição a experiências adversas durante a infância está associada, e é preditora, do índice de sintomas depressivos e tentativas de suicídio durante a idade adulta em mulheres portuguesas. Desse modo, torna-se necessário desenvolver programas de prevenção que levem em consideração a influência das experiências adversas em todo o ciclo vital, especialmente em contextos sociodemográficos mais desfavorecidos.

Apesar de sua contribuição, este estudo tem algumas limitações. Primeiro, é retrospectivo e usa medidas de autorrelato, o que pode ter levado a um viés no sentido de sub ou sobre reportar as experiências de adversidade (Dube et al., 2003; Felitti et al., 1998; Lu et al., 2008). Isso pode decorrer de fatores como estado de humor, problemas de memória e saúde mental (Hardt \& Rutter, 2004) tornando-se pertinente a questão: "o que é relatado é que influencia a psicopatologia ou é o nível de psicopatologia que influencia o que é relatado?". Apesar dessa consideração, um estudo recente e que utilizava o mesmo instrumento (Silva \& Maia, 2013), mostrou que o estado de humor não se relacionava com o número de adversidades relatadas.

Segundo, trata-se de um estudo transversal, o que não permite que se estabeleça relações causais. No entanto, Afifi et al. (2012) sugerem que não experienciar abuso físico traria uma diminuição da incidência de diferentes perturbações, o que decorre da assunção de uma possível relação causal entre abuso físico e perturbações mentais. Terceiro, não foi avaliada a exposição à adversidade na idade adulta. Essa seria uma informação relevante, pois, de acordo com LaNoue et al. (2012), a relação entre adversidade e sintomas depressivos é parcialmente mediada pela vivência de experiências adversas na idade adulta. Quarto, a amostra não é representativa da população portuguesa, não possibilitando, 
desta forma, generalizar os resultados aqui encontrados.

Sugere-se, como investigações futuras, a análise das experiências adversas em diferentes fases de vida e a exploração de diversos fatores que possam ser mediadores (e.g., apoio social, estilo de vinculação, presença de experiências de vida positivas etc.) na relação entre adversidade e psicopatologia.

\section{Referências}

Afifi, T., Boman, J., Fleisher, W., \& Sareen, J. (2009). The relationship between child abuse, parental divorce, and lifetime mental disorders and suicidality in a nationally representative adult sample. Child Abuse \& Neglect, 33(3), 139-147.

Afifi, T., Mota, N., Dasiewicz, P., MacMillan, H., \& Sareen, J. (2012). Physical punishment and mental disorders: Results from a nationally representative US sample. Pediatrics, 130(2), 184-192.

Anda, R., Felitti, V., Bremner, D., Walker, J., Whitfield, C., Perry, B, ... Giles, W. (2006). The enduring effects of abuse and related adverse experiences in childhood: A convergence of evidence from neurobiology and epidemiology. European Archives of Psychiatry and Clinical Neuroscience, 256(3), 174-186.

Azevedo, M., \& Maia, A. (2006). Maus-tratos à criança. Lisboa: Climepsi Editores.

Canavarro, M. (1999). Inventário de sintomas psicopatológicos: B.S.I. In M. Simões, M. Gonçalves, \& L. Almeida (Orgs.), Testes e provas psicológicas em Portugal (Vol.2, pp.87-109). Braga: APPORT/SHO.

Chapman, P., Whitfield, L., Felitti, V., Dube, R., Edwards, J., \& Anda, F. (2004). Adverse childhood experiences and the risk of depressive disorders in adulthood. Journal of Affective Disorders, 82(2), 217-225.

Dube, S., Anda, R., Felitti, V., Chapman, D., Williamson, D., \& Giles, W. (2001). Childhood abuse, household dysfunction, and the risk of attempted suicide throughout the life span: Findings from the adverse childhood experiences study. American Medical Association, 286(24), 3089-3095.

Dube, S., Felitti, V., Dong, M., Giles, W., \& Anda, R. (2003). The impact of adverse childhood experiences on health problems: Evidence from four birth cohorts dating back to 1900. Preventive Medicine, 37(3), 268-277.

Edwards, V., Holden, G., Felitti, V., \& Anda, R. (2003). Relationship between multiple forms of childhood maltreatment and adult mental health in community respondents: Results from the adverse childhood experiences study. American Journal of Psychiatry, 160(8), 1453-1460.
Felitti, V. (2002). The relation between adverse childhood experiences and adult health: Turning gold into lead. The Permanent Journal, 6(1), 44-47.

Felitti, V., Anda, R., Nordenberg, D., Williamson, D., Spitz, A., Edwards, ... Marks, J. (1998). Relationship of childhood abuse and household dysfunction to many of the leading causes of death in adults: The Adverse Childhood Experiences (ACE) study. American Journal of Preventive Medicine, 14(4), 245-258.

Fife-Schaw, C. (2006). Levels of measurement. In G. M. Breakwell, S. Hammond, C. Fife-Schaw, \& J. A. Smith (Eds.), Research methods in Psychology ( $3^{\text {rd }}$ ed.). London: Sage.

Figueiredo, B., Fernandes, E., Matos, R., \& Maia, A. (2002). Maus tratos na infância: trajectórias desenvolvimentais e intervenção psicológica na idade adulta. In R. Abrunhosa \& C. Machado (Coords.), Violência e vítimas de crimes (Vol.1, pp.200-263). Coimbra: Quarteto.

Fuller-Thomson, E., \& Dalton, A. (2011). Suicidal ideation among individuals whose parents have divorced: Findings from a representative Canadian community survey. Psychiatry Research, 187(1-2), 150-155.

Hardt, J., \& Rutter, M. (2004). Validity of adult retrospective reports of adverse childhood experiences: Review of the evidence. Journal of Child Psychology and Psychiatry, 45(2), 260-273.

LaNoue, M., Graeber, D., Hernandez, B., Warner, T., \& Helitzer, D. (2012). Direct and indirect effects of childhood adversity on adult depression. Community Mental Health Journal, 48(2), 187-192.

Larkin, H., Shields, J., \& Anda, R. (2012). The health and social consequences of Adverse Childhood Experiences (ACE) across the lifespan: An introduction to prevention and intervention in the community. Journal of Preventive \& Intervention in the Community, 40(4), 263-270.

Lu, W., Mueser, K., Rosenberg, S., \& Jankowski, M. (2008). Correlates of adverse childhood experiences among adults with severe mood disorders. Psychiatric Services, 59(9), 1018-1026.

Maia, A. (2010). Abordagens neuropsicológicas à vitimação. In C. Machado (Org.), Vitimologia: das novas abordagens teóricas às novas formas de intervenção (pp.81-102). Braga: Psiquilibrios.

MacMillan, H., Fleming, J., Streiner, D., Lin, E., Boyle, M., Jamieson, E. ... Beardslee, W. (2001). Childhood abuse and lifetime psychopathology in a community sample. American Journal of Psychiatry, 158(11), 1878-1883.

Mullen, P., Martin, J., Anderson, J., Romans, S., \& Herbison, G. (1996). The long-term impact of the physical, emotional, and sexual abuse of children: A community study. Child Abuse \& Neglect, 20(1), 7-21.

Nurius, P., Logan-Greene, P., \& Green, S. (2012). Adverse Childhood Experiences (ACE) within a social disadvantage framework: Distinguishing unique, 
cumulative, and moderated contributions to adult mental health. Journal of Prevention \& Intervention in the Community, 40(4), 278-290.

Pinto, R., Correia, L., \& Maia, A. (2014). Assessing the reliability of retrospective reports of adverse childhood experiences among adolescents with documented childhood maltreatment. Journal of Family Violence, 29(4), 431-438. http://dx.doi.org/10.1007/s10896-0 14-9602-9

Rodgers, C., Lang, A., Laffaye, C., Satz, L., Dresselhaus, T., \& Stein, M. (2004). The impact of individual forms of childhood maltreatment on health behavior. Child Abuse \& Neglect, 28(5), 575-586.

Silva, S., \& Maia, A. (2008). Versão portuguesa do Family ACE Questionnaire. In A. Noronha, C. Machado, L. Almeida, M. Gonçalves, S. Martins, \& V. Ramalho (Coords.), Actas da XIII Conferência Internacional de Avaliação Psicológica: Formas e Contextos. Braga: Psiquilibrios.

Silva, S., \& Maia, A. (2010). Experiências adversas na infância e tentativas de suicídio em adultos com obe- sidade mórbida. Revista de Psiquiatria do Rio Grande do Sul, 32(3), 69-72.

Silva, S., \& Maia, A. (2013). The stability of self-reported adverse experiences in childhood: A longitudinal study on obesity. Journal of Interpersonal Violence, 28(10), 1989-2004. http://dx.doi.org/10.1177/0886260512 471077

Whiffen, V., Thompson, J., \& Aube, J., (2000). Mediators of the link between childhood sexual abuse and adult depressive symptoms. Journal of Interpersonal Violence, 15(10), 1100-1120.

Widom, C., Czaja, S., Bentley, T., \& Johnson, M. (2012). A prospective investigation of physical health outcomes in abused and neglect children: New findings from a 30-year follow-up. American Journal of Public Health, 102(6), 1135-1144.

Recebido: fevereiro 20, 2013

Versão final: julho 10, 2013

Aprovado: agosto 28, 2013 
